\title{
Reliability-Based Topology Optimization Using Stochastic Response Surface Method with Sparse Grid Design
}

\author{
Qinghai Zhao, ${ }^{1}$ Xiaokai Chen, ${ }^{1}$ Zheng-Dong Ma, ${ }^{2}$ and Yi Lin ${ }^{3}$ \\ ${ }^{1}$ Collaborative Innovation Center of Electric Vehicles in Beijing, School of Mechanical Engineering, Beijing Institute of Technology, \\ Beijing 100081, China \\ ${ }^{2}$ Department of Mechanical Engineering, University of Michigan, Ann Arbor, MI 48105, USA \\ ${ }^{3}$ Beijing Automotive Technology Center, Beijing 100081, China \\ Correspondence should be addressed to Xiaokai Chen; chenxiaokai@263.net
}

Received 18 July 2014; Revised 11 November 2014; Accepted 27 November 2014

Academic Editor: Chao Jiang

Copyright (C) 2015 Qinghai Zhao et al. This is an open access article distributed under the Creative Commons Attribution License, which permits unrestricted use, distribution, and reproduction in any medium, provided the original work is properly cited.

\begin{abstract}
A mathematical framework is developed which integrates the reliability concept into topology optimization to solve reliability-based topology optimization (RBTO) problems under uncertainty. Two typical methodologies have been presented and implemented, including the performance measure approach (PMA) and the sequential optimization and reliability assessment (SORA). To enhance the computational efficiency of reliability analysis, stochastic response surface method (SRSM) is applied to approximate the true limit state function with respect to the normalized random variables, combined with the reasonable design of experiments generated by sparse grid design, which was proven to be an effective and special discretization technique. The uncertainties such as material property and external loads are considered on three numerical examples: a cantilever beam, a loaded knee structure, and a heat conduction problem. Monte-Carlo simulations are also performed to verify the accuracy of the failure probabilities computed by the proposed approach. Based on the results, it is demonstrated that application of SRSM with SGD can produce an efficient reliability analysis in RBTO which enables a more reliable design than that obtained by DTO. It is also found that, under identical accuracy, SORA is superior to PMA in view of computational efficiency.
\end{abstract}

\section{Introduction}

The subject for optimal structural topologies under uncertainty is very important, challenging, and attractive for researchers. The field of structural topology optimization has become matured since the pioneering work by Bendsøe and Kikuchi [1]. Details of various proposed methodologies can be found in comprehensive reviews and text books [2-4]. The aim of the optimization process is to obtain a material distribution within a fixed design domain, so as to optimize the specified structural response subjected to prescribed constraints. However, most of the optimization problems are established in deterministic manner where the designs are carried out without considering the variations observed in geometry and material property as well as external loads due to inherent uncertainty. Consequently, optimum design obtained by the so-called deterministic topology optimization (DTO) may represent unreasonable reliability level.
Hence, reliability-based topology optimization (RBTO) has emerged which achieves optimal topologies while quantitatively measuring the effects of uncertainty by means of probability constraints.

The state-of-the-art topology optimization methodologies have been applied for obtaining optimized reliable designs of structures and mechanisms considering uncertainties. Density-based method taking into account loading and material uncertainties has been demonstrated for microelectromechanical systems (MEMS) [5], for geometrically nonlinear structures [6]. A level set based approach for compliant mechanisms under uncertainties exhibited by loads, material properties, and member geometries has been reported by Zhang and Ouyang [7]. Recently, topology optimization with uncertainties has been demonstrated for the bidirectional evolutionary structural optimization (BESO) in the design for electrothermal compliant mechanisms [8] and for a vehicle's hood reinforcement [9]. Although the RBTO is a rapidly 
expanding active research field, topology optimization integration with probability constraint is still quite challenging, that is, the numerical difficulty for direct estimation failure probability. Such difficulties have motivated the development of various uncertainty propagation methods, such as MonteCarlo simulation (MCS) method [10], the first and second order reliability methods (FORM/SORM) [11], response surface method (RSM) [12], and the stochastic response surface method (SRSM) [13].

MCS is the most comprehensively used sampling method but the required time and resources can be expensive due to a tremendous number of repeated analyses, especially for general structures with low failure probability. In order to improve efficiency maintaining simplicity, two recommended approaches based on FORM to the failure probability calculation are developed, the reliability index approach (RIA) [14] and its inverse, the more efficient performance measure approach (PMA) [15]. In Maute and Frangopol [5], a robust formulation of RBTO based on the PMA has been presented to account for uncertainties. A comparison study between RIA and PMA has been summarized for RBTO formulation [16], where the results clearly show that PMA has better convergence and efficiency than RIA. In general, the FORMbased RBTO approaches estimate the structure reliability (via reliability index) within the topology optimization algorithm and are referred to as nested-loop (or double-loop) methods. However, empirical evidence suggests that such double-loop approaches lead to substantially high computational cost and weak convergence stability, especially involving virtual simulation models (i.e., finite element models). To overcome these difficulties, some authors proposed different methods to solve RBTO problems seeking for simplification and efficiency formulations. The so-called hybrid (or concurrent) RBTO method has been proposed by Kharmanda and Olhoff [17], where reliability analysis is decoupled from optimization procedures and carried out at the beginning of the optimization loops, followed by equivalent DTO. Luo et al. [18] introduced the fuzzy set theory into multiobjective topology optimization, in which the uncertainties of the objection function and constraints are described by using nonlinear membership functions. A reliability-based topology optimization method using a nonprobabilistic multiellipsoid convex model has been proposed by Luo et al. [19], where the model represents uncertainty of bounded parameters. The theories of a singleloop method for component reliability-based topology optimization (CRBTO) and system reliability-based topology optimization (SRBTO) have been developed by Silva et al. [20] and by Nguyen et al. [21], respectively. The proposed methodology is compatible with existing topology optimization software and suitable for practical applications.

The available reliability analysis using FORM/SORM is conveniently performed with known limit state function. However, in many practical complex structures, the relationship between output response and input date does not exist. Therefore, several surrogate methods, such as the response surface method (RSM) and the Kriging method, are used to approximate the implicit limit state function by a surrogate model. RBTO using standard response surface method has been performed by Yoo et al. [22], where much fewer experimental points are required compared to traditional response surface method. A comparison study between the standard response surface method and Kriging method can be found in Eom et al. [23]. There are not many works focusing on design of experiments (DOE), although it is a key issue for surrogate methods implementation in RBTO.

In this paper, two RBTO methodologies are presented, implemented on several numerical examples, including performance measure approach (PMA) and sequential optimization and reliability assessment (SORA). Topology optimization method is solved by the solid isotropic microstructure with penalty (SIMP) method. Reliability analysis is carried out by FORM, where the limit state function is approximated by the stochastic response surface method (SRSM). Design of experiment (DOE) is generated by sparse grid design (SGD), which was proven to be an effective and special discretization technique. The proposed approaches are capable of solving a wide range of problems, but the focus of this paper is to be limited to minimum-volume optimization problems for a detailed description. Monte-Carlo simulations are also performed to verify the accuracy of the failure probabilities computed by the proposed approach.

The paper is organized as follows. In Section 2, the basic formulation of RBTO is presented, followed by the selected methodologies including PMA and SORA. Descriptions of the SRSM and SGD are briefly discussed in Sections 3 and 4, respectively. The flowchart and procedure of PMA and SORA are presented in Section 5. Numerical results and discussion for RBTO methodologies and DTO are illustrated in Section 6. Finally, the conclusion of the paper is provided in Section 7.

\section{Reliability-Based Topology Optimization}

In general, RBTO problems are formulated as follows:

$$
\begin{aligned}
\min _{\boldsymbol{\rho}} & C(\boldsymbol{\rho}) \\
\text { s.t. } & \operatorname{Pr}\left[G_{i}(\boldsymbol{\rho}, \mathbf{X}) \leq 0\right] \leq P_{f_{i}}^{T} \quad i=1, \ldots, m \\
& \boldsymbol{\rho}_{\min } \leq \boldsymbol{\rho} \leq \boldsymbol{\rho}_{\max },
\end{aligned}
$$

where $\rho$ is the vector of design variables (i.e., the element densities), defined as independent deterministic variables with the upper bounds $\boldsymbol{\rho}_{\max }$ and lower bounds $\boldsymbol{\rho}_{\min }, \mathbf{X}$ is the vector of random variables (i.e., loads and material property) with the realizations being noted $\mathbf{x}, C(\cdot)$ is the objective function, $G_{i}(\cdot), i=1, \ldots, m$, is the $i$ th limit state function or performance function, $\operatorname{Pr}[\cdot]$ is the probability operator, $P_{f_{i}}^{T}$ is the target failure probability for the $i$ th constraint, and $m$ is the number of probabilistic constraints. To evaluate the failure probability, the safe region is defined as $G_{i}(\boldsymbol{\rho}, \mathbf{X})>0$, the failure region as $G_{i}(\boldsymbol{\rho}, \mathbf{X})<0$, and the limit state function as $G_{i}(\boldsymbol{\rho}, \mathbf{X})=0$. The failure probability for each constraint may be obtained by evaluating the multidimensional integral as follows:

$$
P_{f_{i}}=\operatorname{Pr}\left[G_{i}(\boldsymbol{\rho}, \mathbf{X}) \leq 0\right]=\int \cdots \int_{G_{i}(\boldsymbol{\rho}, \mathbf{X}) \leq 0} f_{\mathbf{X}}(\mathbf{x}) d \mathbf{x},
$$


where $P_{f_{i}}$ is the failure probability and $f_{\mathbf{X}}(\mathbf{x})$ is the joint probability density function (PDF) of random variables $\mathbf{X}$. In practice, it is difficult and even impossible to compute this integral exactly. In this paper, FORM is adopted for reliability analysis because of its efficiency and simplicity. The key concept of FORM is to find the most probable point (MPP), which is defined as the point on the limit state function closest to the origin in the normalized space transformed from the physical space through the Rosenblatt or the Nataf transformations. By FORM approximation, the failure probability and the allowable failure probability are evaluated by

$$
P_{f_{i}}^{T} \approx \Phi\left(-\beta_{i}^{T}\right), \quad P_{f_{i}} \approx \Phi\left(-\beta_{i}\right),
$$

where $\Phi(\cdot)$ is the standard cumulative distribution function (CDF) and $\beta_{i}^{T}$ is the target reliability index for the $i$ th constraint and the reliability index $\beta$ [24], which is a reliability measurement defined as the distance between the MPP and the origin. By applying the Rosenblatt or the Nataf transformations, random vector $\mathbf{X}$ is transformed to a vector of normalized and independent Gaussian $\mathbf{U}$ (zero means and unit variance), expressed as $\mathbf{U}=T(\mathbf{X})$ or $\mathbf{X}=T^{-1}(\mathbf{U})$. Based on the above transformation, the limit state function is correspondingly defined as

$$
G_{i}(\boldsymbol{\rho}, \mathbf{X})=G_{i}\left(\boldsymbol{\rho}, T^{-1}(\mathbf{U})\right)=g_{i}(\boldsymbol{\rho}, \mathbf{U}),
$$

where $g_{i}$ is the $i$ th limit state function in the normalized space.

2.1. Performance Measure Approach (PMA) for RBTO. In this paper, PMA is used as a reference comparison method for SORA. By using PMA formulation, the RBTO is expressed as

$$
\begin{array}{ll}
\min _{\boldsymbol{\rho}} & C(\boldsymbol{\rho}) \\
\text { s.t. } & G_{i}^{p}\left(\boldsymbol{\rho}, \mathbf{x}_{i}^{*}\right) \geq 0 \quad i=1, \ldots, m \\
& \boldsymbol{\rho}_{\min } \leq \boldsymbol{\rho} \leq \boldsymbol{\rho}_{\max },
\end{array}
$$

where $G_{i}^{p}$ is the target performance with respect to the $i$ th limit state function calculated at the MPP denoted as $\mathbf{x}_{i}^{*}$ in physical space or $\mathbf{u}_{i}^{*}$ in normalized space, which can be found by the inverse reliability analysis, given as

$$
\begin{array}{ll}
\min _{\mathbf{u}} & g_{i}(\mathbf{u}) \\
\text { s.t. } & \|\mathbf{u}\|=\beta_{i}^{T} .
\end{array}
$$

As the inverse reliability analysis is an optimization procedure by itself, the RBTO is a typical double-loop strategy, where the outer loop is an optimization problem in terms of design variables $\rho$ and the inner loop for reliability analysis in terms of random variables $\mathbf{X}$.

2.2. Sequential Optimization and Reliability Assessment (SORA) for RBTO. Initiated by $\mathrm{Du}$ and Chen [25], this approach decouples the RBTO model into a series of cycles of DTO and reliability analysis. In each cycle, DTO and reliability analysis are decoupled from each other and reliability analysis is only conducted after the DTO. SORA for RBTO can be expressed as

$$
\begin{array}{ll}
\min _{\boldsymbol{\rho}} & C\left(\boldsymbol{\rho}^{k}\right) \\
\text { s.t. } & G_{i}\left(\boldsymbol{\rho}^{k}, \mathbf{x}_{i}^{k-1}\right) \geq 0 \quad i=1, \ldots, m \\
& \boldsymbol{\rho}_{\min } \leq \boldsymbol{\rho}^{k} \leq \boldsymbol{\rho}_{\max },
\end{array}
$$

where $k$ indicates the end cycle of each deterministic topology optimization and $\mathbf{x}_{i}^{k-1}$ is the MPP in physical space with respect to the $i$ th limit state in the $(k-1)$ th cycle, given by the transformation of $\mathbf{u}_{i}^{*(k-1)}$. By the given current optimal $\operatorname{design} \boldsymbol{\rho}^{*(k)}$, the next MPP $\mathbf{u}_{i}^{*(k)}$ is obtained by the inverse reliability analysis, defined as

$$
\begin{array}{ll}
\min _{\mathbf{u}} & g_{i}\left(\boldsymbol{\rho}^{*(k)}, \mathbf{u}\right) \\
\text { s.t. } & \|\mathbf{u}\|=\beta_{i}^{T} .
\end{array}
$$

The advantage of the SORA formulation is that the number of reliability analyses will be significantly reduced as it is equal to the number of cycles. The entire optimization process is repeated until the deterministic topology optimization becomes convergent and reliability requirements are satisfied.

\section{Stochastic Response Surface Method}

The stochastic response surface method (SRSM) is the most widely used surrogate method in structural reliability analysis, which can be viewed as a conceptual extension of classical deterministic response surface method [26]. The SRSM approximates the true limit state function through series expansions of standard random variables which is typically a $k$ th order Hermite orthogonal polynomial with undetermined coefficients. Consider

$$
\begin{aligned}
g(\mathbf{u})= & a_{0} \Gamma_{0}+\sum_{i_{1}=1}^{d} a_{i_{1}} \Gamma_{1}\left(u_{i_{1}}\right) \\
& +\sum_{i_{1}=1}^{d} \sum_{i_{2}=1}^{i_{1}} a_{i_{1} i_{2}} \Gamma_{2}\left(u_{i_{1}}, u_{i_{2}}\right) \\
& +\sum_{i_{1}=1}^{d} \sum_{i_{2}=1}^{i_{1}} \sum_{i_{3}=1}^{i_{2}} a_{i_{1} i_{2} i_{3}} \Gamma_{3}\left(u_{i_{1}}, u_{i_{2}}, u_{i_{3}}\right)+\cdots
\end{aligned}
$$

where $\left\{u_{i}\right\}_{i=1}^{\infty}$ is a set of independent normalized random variables, $a_{0}, a_{i_{1}}, \ldots$ are deterministic coefficients to be estimated, $g(\mathbf{u})$ is the approximated limit state function, and $\Gamma_{i}$ are Hermite polynomials of degree $p$, given by

$$
\Gamma_{p}\left(u_{i_{1}}, \ldots, u_{i_{p}}\right)=(-1)^{p} e^{1 / 2} \mathbf{u}^{T} \mathbf{u} \frac{\partial^{P}}{\partial u_{i_{1}} \cdots \partial u_{i_{p}}} e^{-1 / 2} \mathbf{u}^{T} \mathbf{u}
$$


where $\mathbf{u}$ is the vector of $p$ independent normal random variables $\left\{u_{i}\right\}_{i=1} p$, which represent the input uncertainties. Furthermore, the Hermite polynomials defined on $\left\{u_{i}\right\}_{i=1} p$ are orthogonal with respect to an inner product defined as the expectation of the product of two random variables [13]. Thus,

$$
E\left\lfloor\Gamma_{p} \Gamma_{q}\right\rfloor=0 \quad \text { iff } \Gamma_{p} \neq \Gamma_{q} .
$$

Applying the least square method, the coefficients $\mathbf{b}=$ $\left[a_{0}, a_{i_{1}}, \ldots, a_{i_{p}}\right]$ can be calculated by minimizing the square of error expressed as follows:

$$
\min J(\mathbf{b})=\|\overline{\mathbf{g}}-\mathbf{A b}\|^{2},
$$

where $\overline{\mathbf{g}}(\mathbf{u})$ is the corresponding real function evaluated at a set of sampling points. The analytical solution can be expressed as

$$
\mathbf{b}=\left(\mathbf{A}^{T} \mathbf{A}\right)^{-1} \mathbf{A}^{T} \overline{\mathbf{g}}
$$

where $\mathbf{A}$ is the matrix of bases at the sampling points, defined as

$$
\mathbf{A}=\left[\begin{array}{cccc}
\Gamma_{0}\left(u_{1}\right) & \Gamma_{1}\left(u_{1}\right) & \cdots & \Gamma_{P}\left(u_{1}\right) \\
\Gamma_{0}\left(u_{2}\right) & \Gamma_{1}\left(u_{2}\right) & \cdots & \Gamma_{P}\left(u_{2}\right) \\
\vdots & \vdots & \ddots & \vdots \\
\Gamma_{0}\left(u_{N}\right) & \Gamma_{1}\left(u_{N}\right) & \cdots & \Gamma_{P}\left(u_{N}\right)
\end{array}\right]
$$

Therefore, one key issue when applying SRSM for structural reliability analysis is to choose a suitable design of experiment to generate sampling points.

\section{Sparse Gird Design}

The sparse grid design is a special discretization technique, which can be traced back to the Smolyak algorithm [27]. It is based on hierarchical basis, a representation of a discrete function space which is equivalent to the conventional nodal basis, and a sparse tensor product construction [28]. The onedimensional difference quadrature formula is defined as

$$
\Delta_{k}^{1}(f)=\left(Q_{k}^{1}-Q_{k-1}^{1}\right)(f), \quad \text { with } Q_{0}^{1}(f)=0 .
$$

Then, the Smolyak quadrature formula for $d$-dimensional functions $f$ with level $l \in N$ is given by

$$
Q_{l}^{d}(f)=\sum_{|\mathbf{k}| \leq l+d}\left(\Delta_{k_{1}}^{1} \otimes \cdots \otimes \Delta_{k_{d}}^{1}\right)(f) .
$$

Note that $|\mathbf{k}|$ denotes the summation of the multi-indices $\left(|\mathbf{k}|=k_{1}+\cdots+k_{d}\right)$. Alternatively, the above formula can be written as

$$
\begin{aligned}
Q_{l}^{d}(f)= & \sum_{l+1 \leq|\mathbf{k}| \leq l+d}(-1)^{l+d-|\mathbf{k}|} \\
& \cdot\left(\begin{array}{c}
d-1 \\
l+d-|\mathbf{k}|
\end{array}\right) \cdot\left(Q_{k_{1}}^{1} \otimes \cdots \otimes Q_{k_{d}}^{1}\right)(f) .
\end{aligned}
$$

To compute $Q_{l}^{d}(f)$, specific tensor product rules of sparse grid are needed, defined as

$$
U_{l}^{d}=\bigcup_{l+1 \leq|\mathbf{k}| \leq l+d}\left(U_{k_{1}}^{1} \otimes \cdots \otimes U_{k_{d}}^{1}\right)
$$

where $U_{1}^{i}$ denote the one-dimension support nodes, which can be obtained by univariate quadrature algorithms, such as Newton-Cotes, Gauss quadrature, and Clenshaw-Curtis rules [29].

In this paper, the Clenshaw-Curtis type sparse grid design $H^{\mathrm{CC}}$ is constructed, with equidistant nodes for polynomial interpolation. Here, the $u_{k}^{i}$ are defined as

$$
u_{k}^{i}= \begin{cases}\frac{(i-1)}{\left(m_{k}-1\right)} & \text { for } i=1, \ldots, m_{k}, \text { if } m_{k}>1 \\ 0.5 & \text { for } i=1, \text { if } m_{k}=1\end{cases}
$$

where

$$
m_{k}= \begin{cases}1 & \text { if } k=1 \\ 2^{k-1}+1 & \text { if } k>1\end{cases}
$$

Figure 1 illustrates the grids $H^{\mathrm{CC}}$ obtained using the Clenshaw-Curtis rule of dimensions $d=2$ and $d=3$ with level $l=2$.

\section{Flowchart and Procedure of RBTO}

The flowcharts of the RBTO/PMA and RBTO/SORA methods are provided in Figure 2. For example, the strategy of SORA contains two parts: "equivalent" deterministic optimization and independent reliability analysis. At first, the topology optimization problem is formulated based on SIMP method and solved by using the method of moving asymptotes (MMA) with standard settings [30]. To ensure manufacturability and mesh independence, the mesh-independent density filtering is applied to eliminate the appearance of numerical instabilities [31]. Then, the reliability analysis is implemented at the deterministic optimum solution to locate the MPP that satisfies the desired target reliability index, where the SRSM approximates the true limit state function by regression analysis of the least square method, combined with SGM adopted to generate the experimental sample points based on random variables. The new deterministic optimization model is constructed based on the MPP while the stop criterion is not satisfied. Next procedure cycle is running until the stopping criterion is convergent.

\section{Numerical Examples}

6.1. The $M B B$ Beam. In the first example, consider the popular MBB beam depicted in Figure 3. The dimensions of the beam are $L=90 \mathrm{~mm}$ and $H=30 \mathrm{~mm}$ and the thickness is $t=1 \mathrm{~mm}$. Young's modulus $E_{0}=2.10 \times 10^{5} \mathrm{MPa}$ and Poisson's ratio $v=0.30$ are assumed. An external load $F=100 \mathrm{~N}$ is applied. Design domain is discretized by $2700(90 \times 30)$ fournode bilinear finite elements. Random variables are chosen 


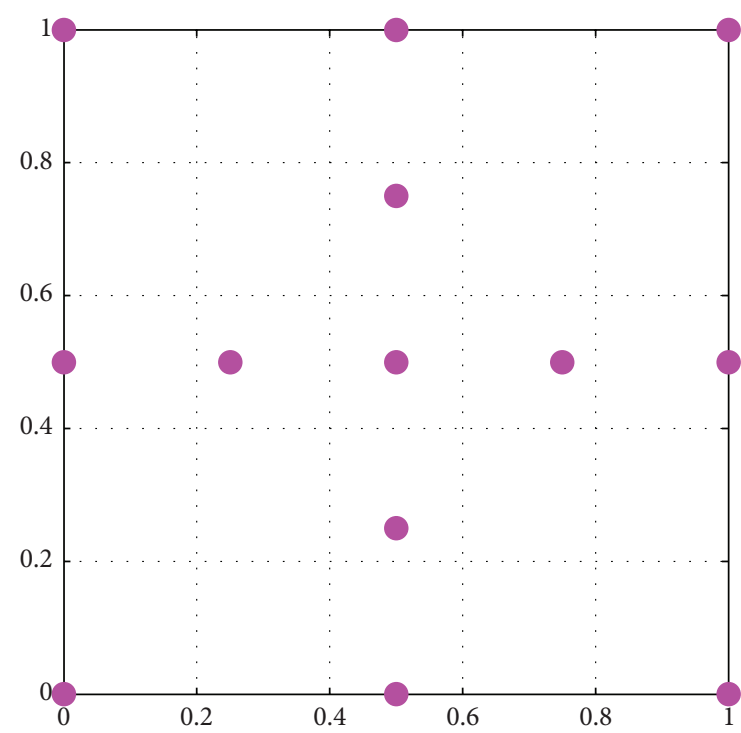

(a) $d=2, l=2$, points: 13

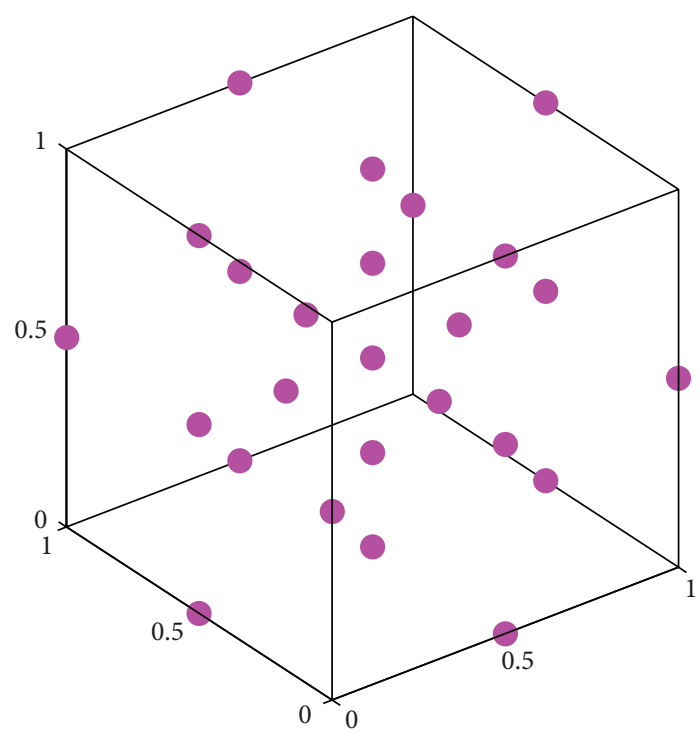

(b) $d=3, l=2$, points: 25

FIGURE 1: The Clenshaw-Curtis type sparse grid design.

TABLE 1: Comparison of topology optimization design results.

\begin{tabular}{lcccc}
\hline Approach & Volume/volume fraction $\left(\mathrm{mm}^{2}\right) / \%$ & Reliability index $(\beta)$ & Computing time $(\mathrm{s})$ & \multicolumn{2}{c}{ Design point } & $E_{0} / \mathrm{MPa}$ & - \\
& & & 314.74 & - \\
\hline DTO & $1047.19 / 38.78$ & - & 1655.11 & $1.59 \times 10^{5}$ \\
PMA & $1351.48 / 50.05$ & 3.002 & 493.58 & 117.68 \\
SORA & $1351.48 / 50.05$ & 3.002 & $1.59 \times 10^{5}$ & 117.68 \\
\hline
\end{tabular}

as Young's modulus $E_{0}$ and external load $F$. Each variable is assumed to be normally distributed and has $10 \%$ standard deviation of the mean value.

RBTO problems are formulated as follows:

$$
\begin{array}{ll}
\min _{\boldsymbol{\rho}} & V \\
\text { s.t. } & \operatorname{Pr}[G(\boldsymbol{\rho}, \mathbf{X}) \leq 0] \leq P_{f}^{T}=0.135 \% \\
& \boldsymbol{\rho}_{\text {min }} \leq \boldsymbol{\rho} \leq \boldsymbol{\rho}_{\text {max }},
\end{array}
$$

where $V$ is the volume and the limit state function is defined as $G=\delta_{\max }-\delta . \delta$ is the actual displacement and $\delta_{\max }$ is the allowable displacement assigned as $0.140 \mathrm{~mm}$. The probability of constraint violation is less than $0.135 \%$, which means that the target reliability index is equal to 3.0.

The optimal topologies obtained by DTO and RBTO approaches are presented in Figures 4 and 5, respectively. The optimization results obtained from each approach are summarized in Table 1, including volume/volume fraction, reliability index, computing time, and design point. The reliability index is calculated by 10,000 Monte-Carlo simulations. And the coefficients of SRSM to construct limit state functions at some iteration during PMA and SORA procedures are given in Tables 2 and 3 , respectively, where $u_{1}$ and $u_{2}$ are the normalized values of Young's modulus $E_{0}$ and applied load $F$, respectively.
TABLE 2: Approximated limit state function constructed by SRSM in PMA.

\begin{tabular}{lcccccc}
\hline Iteration & $a_{0}$ & $a_{1}$ & $a_{2}$ & $a_{3}$ & $a_{4}$ & $a_{5}$ \\
\hline 1 & -2.5006 & 0.3782 & -0.3661 & -0.0381 & 0.0381 & -0.0004 \\
2 & -0.3879 & 0.1500 & -0.1451 & -0.0151 & 0.0151 & -0.0001 \\
3 & 0.2686 & 0.0790 & -0.0765 & -0.0080 & 0.0080 & -0.0001 \\
$\vdots$ & $\vdots$ & $\vdots$ & $\vdots$ & $\vdots$ & $\vdots$ & $\vdots$ \\
101 & 0.2484 & 0.0812 & -0.0786 & -0.0082 & 0.0082 & -0.0001 \\
102 & 0.2484 & 0.0812 & -0.0786 & -0.0082 & 0.0082 & -0.0001 \\
103 & 0.2484 & 0.0812 & -0.0786 & -0.0082 & 0.0082 & -0.0001 \\
\hline
\end{tabular}

Approximated limit state function: $g(\mathbf{u})=a_{0}+a_{1} u_{1}+a_{2} u_{2}+a_{3}\left(u_{1}^{2}-1\right)+$ $a_{4} u_{1} u_{2}+a_{5}\left(u_{2}^{2}-1\right)$.

TABLE 3: Approximated limit state function constructed by SRSM in SORA.

\begin{tabular}{lcccccc}
\hline Iteration & $a_{0}$ & $a_{1}$ & $a_{2}$ & $a_{3}$ & $a_{4}$ & $a_{5}$ \\
\hline 1 & -0.0102 & 0.1092 & -0.1057 & -0.0110 & 0.0110 & -0.0001 \\
2 & 0.2484 & 0.0812 & -0.0786 & -0.0082 & 0.0082 & -0.0001 \\
\hline
\end{tabular}

From the comparison of the results, the optimal solutions obtained by RBTOs have different topologies from those obtained by DTO with the application of SRSM with SGD in reliability analysis, as shown in Figures 4 and 5. As the results listed in Table 1 show, RBTOs use more material than DTO by 


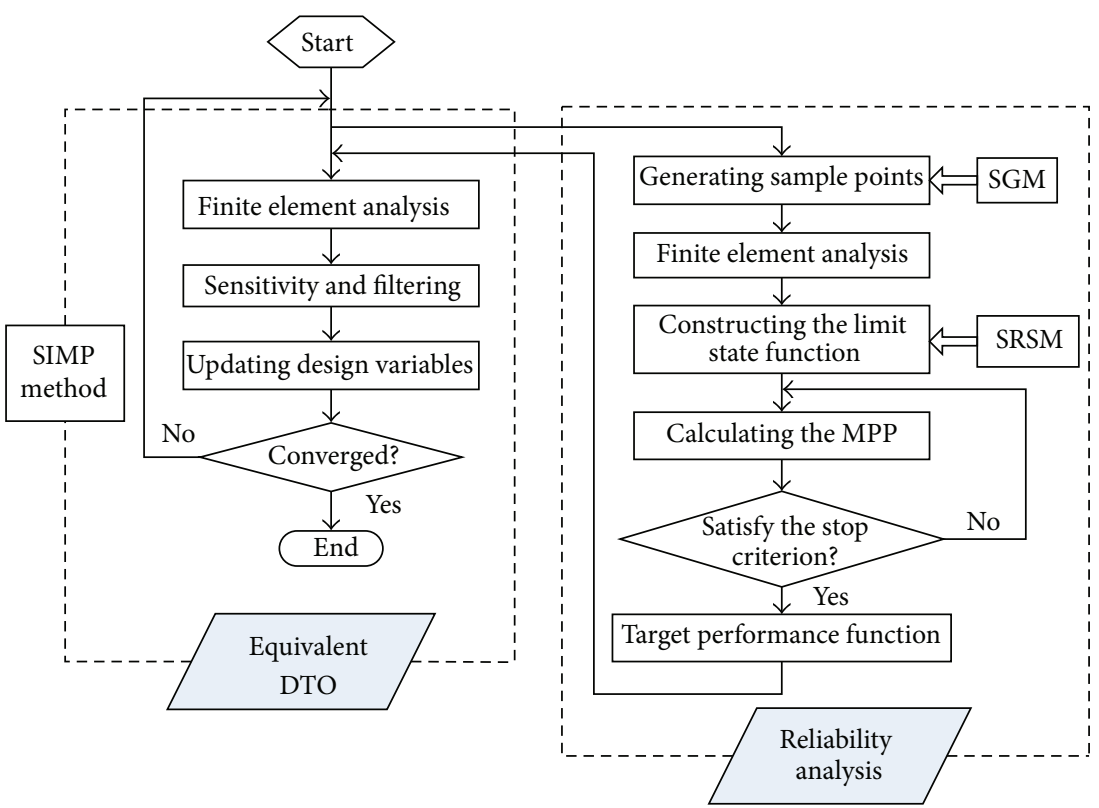

(a) PMA

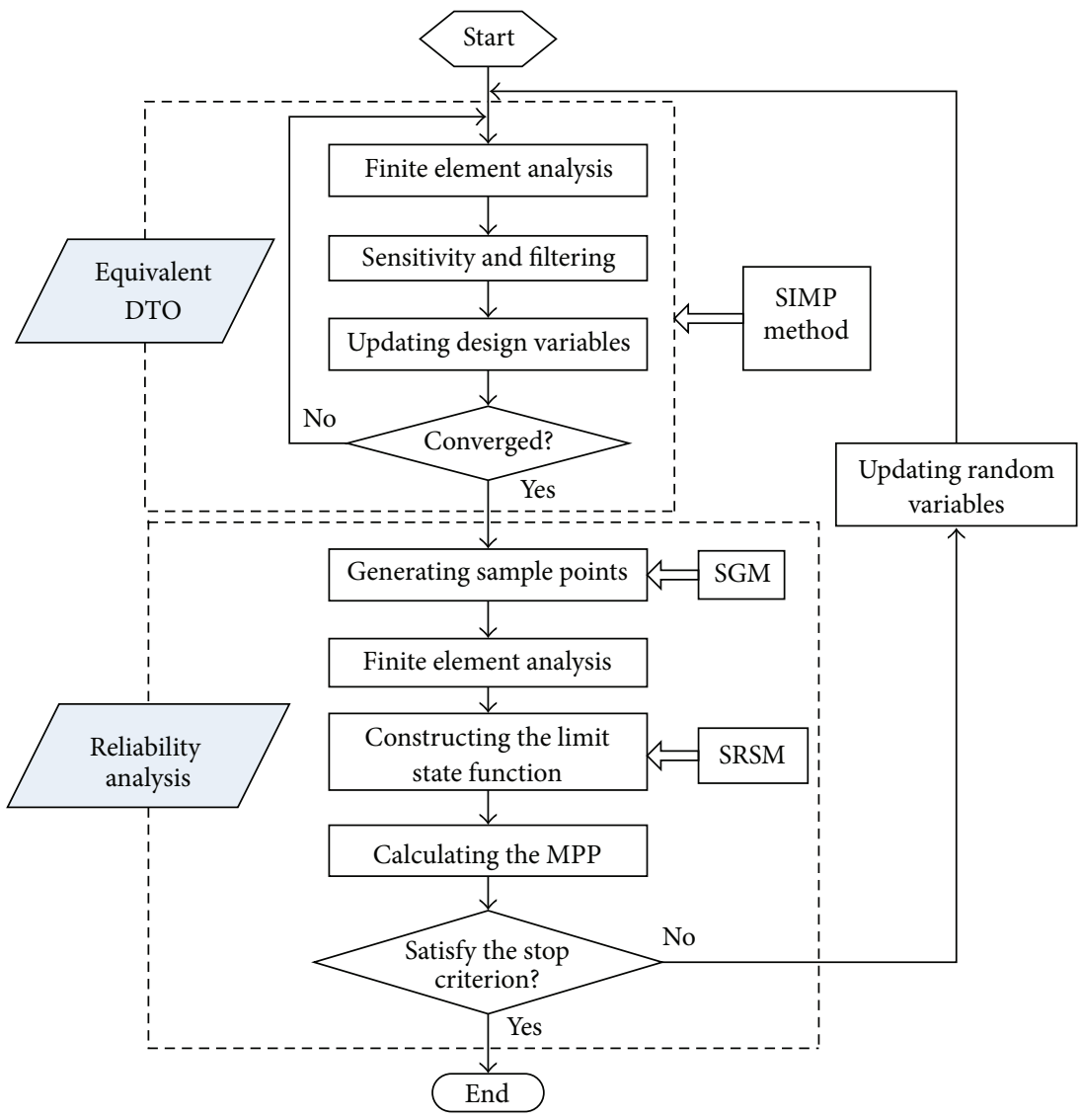

(b) SORA

FIgURE 2: The flowchart of RBTO. 
TABLE 4: Comparison of topology optimization design results.

\begin{tabular}{|c|c|c|c|c|c|c|}
\hline \multirow{2}{*}{ Approach } & \multirow{2}{*}{ Volume/volume fraction $\left(\mathrm{mm}^{2}\right) / \%$} & \multirow{2}{*}{ Reliability index $(\beta)$} & \multirow{2}{*}{ Computing time (s) } & \multicolumn{3}{|c|}{ Design point } \\
\hline & & & & $E_{0} / \mathrm{MPa}$ & $F_{x} / \mathrm{N}$ & $F_{y} / \mathrm{N}$ \\
\hline DTO & $918.59 / 25.52$ & - & 971.31 & - & - & - \\
\hline PMA & $1345.49 / 37.37$ & 3.003 & 8266.06 & $5.62 \times 10^{4}$ & 171.53 & 116.15 \\
\hline SORA & $1345.49 / 37.37$ & 3.003 & 2296.5 & $5.62 \times 10^{4}$ & 171.53 & 116.15 \\
\hline
\end{tabular}

TABLE 5: Approximated limit state function constructed by SRSM in PMA.

\begin{tabular}{lcccccccccc}
\hline Iteration & $a_{0}$ & $a_{1}$ & $a_{2}$ & $a_{3}$ & $a_{4}$ & $a_{5}$ & $a_{6}$ & $a_{7}$ & $a_{8}$ & $a_{9}$ \\
\hline 1 & -1.9135 & 0.3228 & -0.2885 & -0.3034 & -0.0326 & 0.0306 & -0.0144 & 0.0000 & -0.0152 & 0.0322 \\
2 & -0.2137 & 0.1345 & -0.1099 & -0.1366 & -0.0136 & 0.0117 & -0.0055 & 0.0000 & -0.0068 & 0.0145 \\
$\vdots$ & $\vdots$ & $\vdots$ & $\vdots$ & $\vdots$ & $\vdots$ & $\vdots$ & $\vdots$ & $\vdots$ & $\vdots$ \\
170 & 0.3517 & 0.0718 & -0.0624 & -0.0693 & -0.0073 & 0.0066 & -0.0031 & -0.0000 & -0.0035 & 0.0073 \\
171 & 0.3517 & 0.0718 & -0.0624 & -0.0693 & -0.0073 & 0.0066 & -0.0031 & -0.0000 & -0.0035 & 0.0073 \\
\hline
\end{tabular}

Approximated limit state function: $g(\mathbf{u})=a_{0}+a_{1} u_{1}+a_{2} u_{2}+a_{3} u_{3}+a_{4}\left(u_{1}^{2}-1\right)+a_{5} u_{1} u_{2}+a_{6}\left(u_{2}^{2}-1\right)+a_{7} u_{2} u_{3}+a_{8}\left(u_{3}^{2}-1\right)+a_{9} u_{1} u_{3}$.

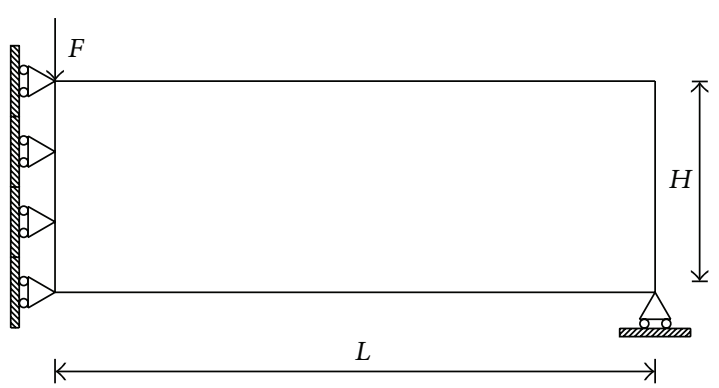

FIgure 3: The design domain of the MBB beam.

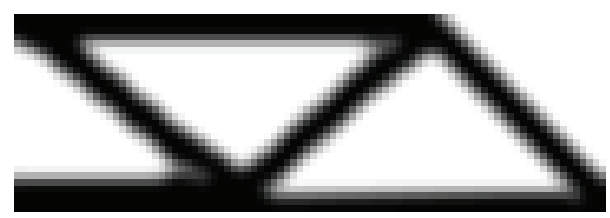

FIGURE 4: Resulting topology of DTO.

$11.27 \%$, improving the reliability index up to 3.002. It can also be seen from the results listed in Table 1 that SORA seems to be more efficient than PMA, with the evidence in computing time and the number of reliability analyses, as presented in Tables 1, 2, and 3 .

6.2. The Loaded Knee Structure. Next, the loaded knee structures with multiple load cases are depicted in Figure 6. The dimensions of the structure are $L=60 \mathrm{~mm}$ and the thickness is $t=1 \mathrm{~mm}$. Young's modulus $E_{0}=7.10 \times 10^{4} \mathrm{MPa}$ and Poisson's ratio $v=0.33$ are assumed. Two external load cases, $F_{x}=150 \mathrm{~N}$ and $F_{y}=100 \mathrm{~N}$, are applied on the midpoint of the right edge, respectively. Design domain is discretized by $3600(60 \times 60)$ four-node elements. Random variables are chosen as Young's modulus $E_{0}$ and external loads $F_{x}$ and $F_{y}$. Each variable is assumed to be normally distributed and has
$10 \%$ standard deviation of the mean value. It should be noted that sampling points generated by SGD are extended to three dimensions in this example.

In this example, the objective function is volume, and the limit state function is defined as $G=c_{\max }-c$. $c$ is the sum of compliance for the two load cases, and $c_{\max }$ is the allowable compliance and is assigned to $40 \mathrm{~N} \cdot \mathrm{mm}$. The target reliability index is set to 3.0 .

The optimal topologies obtained by DTO and RBTO approaches are presented in Figures 7 and 8, respectively. The optimization results obtained from each approach are summarized in Table 4, including volume/volume fraction, reliability index, computing time, and design point. The reliability index is calculated by Monte-Carlo simulations. And the coefficients of SRSM to construct limit state functions at some iteration during PMA and SORA procedures are given in Tables 5 and 6 , respectively, where $u_{1}$ and $u_{2}$ are the normalized values of Young's modulus $E_{0}$ and applied loads $F_{x}$ and $F_{y}$, respectively.

From the comparison of the results, the application of SRSM with SGD in RBTO enables different topologies from that obtained by DTO, as shown in Figures 7 and 8. RBTOs use more material by $11.85 \%$ than DTO, corresponding with the reliability index rising to 3.003 , as the results listed in Table 4. It can also be seen from the results listed in Table 4 that there is practically no difference in the optimum volume obtained by SORA and PMA. However, the number of reliability analyses for SORA significantly reduces to 3, compared with that for PMA which rises to 171 . Based on the result, it also concluded that SRSM has good performance in reliability analysis and that SGD is able to generate reasonable sampling points.

6.3. Heat Conduction Problem. Here, the extended heat conduction problem is considered, as shown in Figure 9. The dimensions of the structure are $L=80 \mathrm{~mm}$ and the thickness is $t=1 \mathrm{~mm}$. The thermal conductivity $k_{0}=0.4 \mathrm{~W} /(\mathrm{mm} \cdot \mathrm{K})$ is 


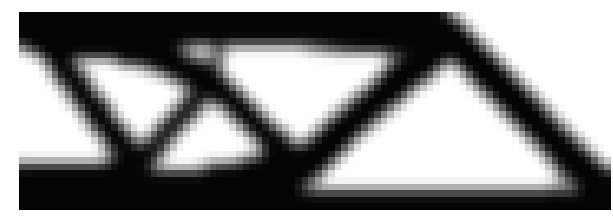

(a)

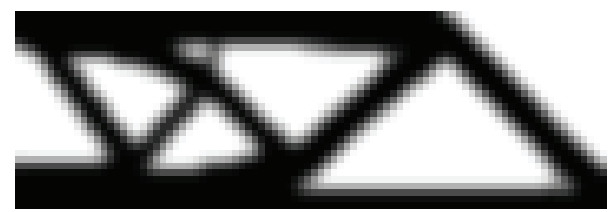

(b)

Figure 5: Resulting topologies of RBTO: (a) PMA; (b) SORA.

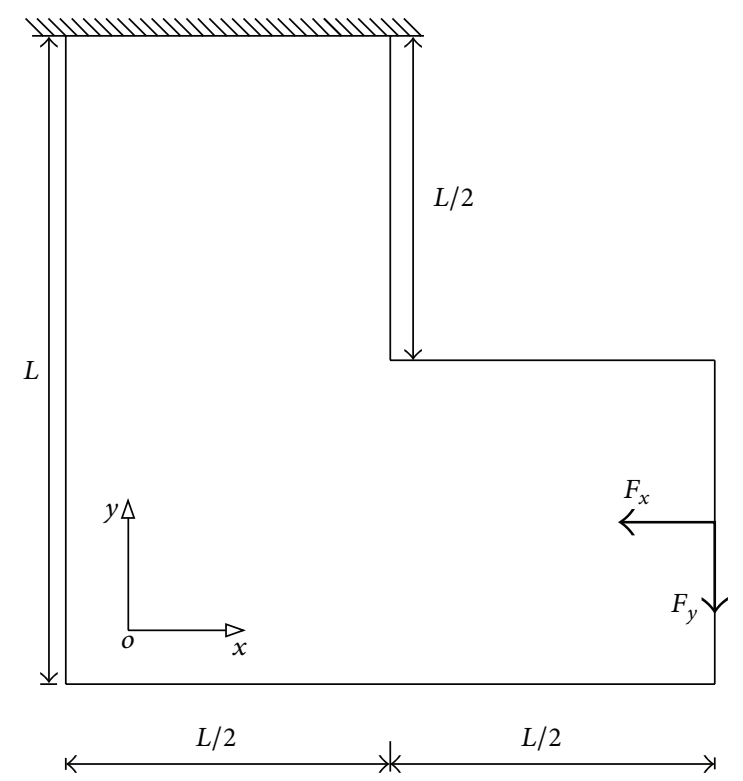

FIgURE 6: Design domain of the loaded knee structure.

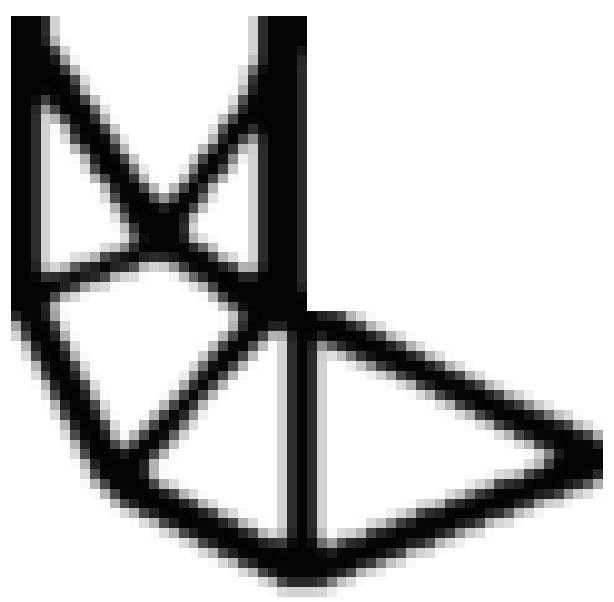

Figure 7: Resulting topology of DTO.

TABLE 6: Approximated limit state function constructed by SRSM in SORA.

\begin{tabular}{lcccccccccc}
\hline Iteration & $a_{0}$ & $a_{1}$ & $a_{2}$ & $a_{3}$ & $a_{4}$ & $a_{5}$ & $a_{6}$ & $a_{7}$ & $a_{8}$ & $a_{9}$ \\
\hline 1 & -0.0199 & 0.1130 & -0.0971 & -0.1101 & -0.0114 & 0.0103 & -0.0048 & -0.0000 & -0.0055 & 0.0117 \\
2 & 0.3531 & 0.0717 & -0.0623 & -0.0691 & -0.0072 & 0.0066 & -0.0031 & 0.0000 & -0.0035 & 0.0073 \\
3 & 0.3517 & 0.0718 & -0.0624 & -0.0693 & -0.0073 & 0.0066 & -0.0031 & -0.0000 & -0.0035 & 0.0073 \\
\hline
\end{tabular}




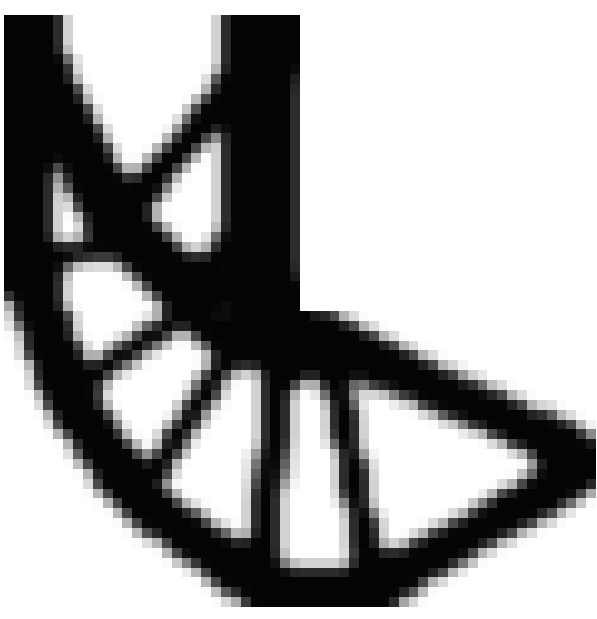

(a)

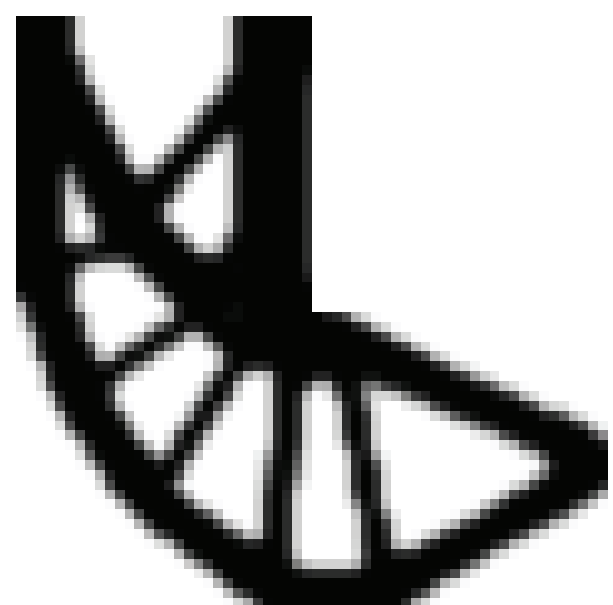

(b)

Figure 8: Resulting topologies of RBTO: (a) PMA; (b) SORA.

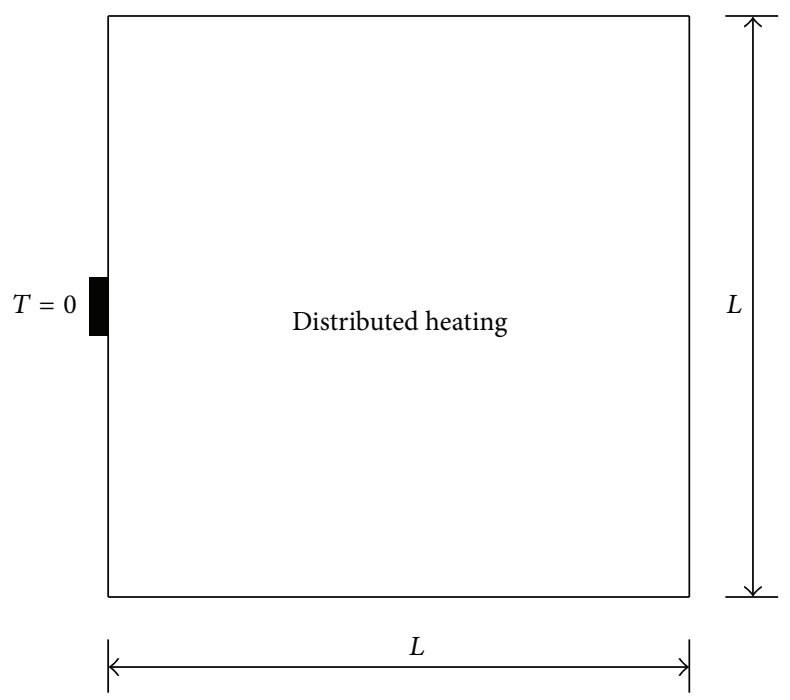

FIGURE 9: Design domain of the loaded knee structure.

assumed. The square plate is evenly heated by constant heat source $Q=0.001 \mathrm{~W} / \mathrm{mm}^{2}$ and the temperature of the center of the left edge is set to 0 . Design domain is discretized by $6400(80 \times 80)$ four-node elements. Random variables are chosen as thermal conductivity $k_{0}$ and constant heat source $Q$. Each variable is assumed to be normally distributed and has $10 \%$ standard deviation of the mean value.

In this example, the objective function is volume, and the limit state function is defined as $G=\varphi_{\max }-\varphi \cdot \varphi$ is the dissipation of heat transport potential capacity, and $\varphi_{\max }$ is the allowable dissipation of heat transport potential capacity and is assigned to $500 \mathrm{~W}$. The target reliability index is set to 3.0.

The optimal topologies and temperature contours obtained by DTO and RBTOs are presented in Figures 10 and 11, respectively. The optimization results obtained from each approach are summarized in Table 7, including volume/volume fraction, reliability index, computing time, and design point. The reliability index is calculated by Monte-Carlo simulations. And the coefficients of SRSM to construct limit state functions at some iteration during PMA and SORA procedures are given in Tables 8 and 9, respectively, where $u_{1}$ and $u_{2}$ are the normalized values of thermal conductivity $k_{0}$ and constant heat source $Q$, respectively.

It can be found that the application of SRSM with SGD in RBTO also has well-behaved heat conduction problem. From the comparison of the results, the optimal solutions obtained by RBTO have different topologies from those obtained by DTO, as shown in Figure 10. As the results listed in Table 7 show, RBTOs use more material than DTO by $6.84 \%$, improving the reliability index by up to 3.003 . It can also be seen from the results listed in Table 7 that the optimum volume obtained by SORA and PMA is identical. However, SORA seems to be more efficient than PMA, as the number of reliability analyses significantly reduces, as presented in Tables 8 and 9. 


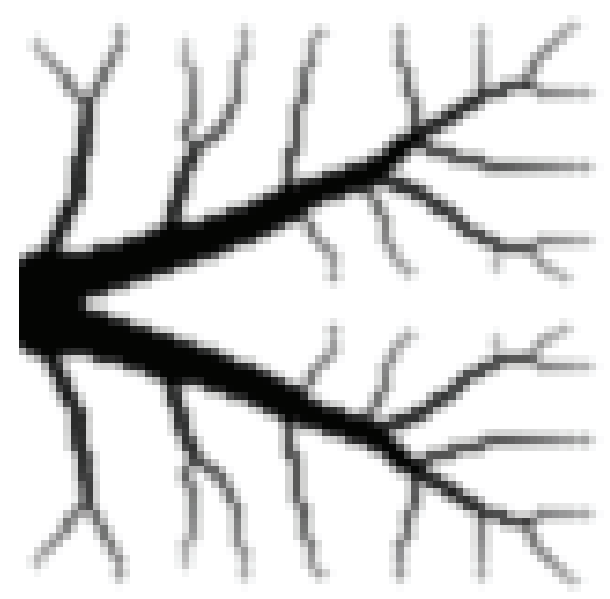

(a)

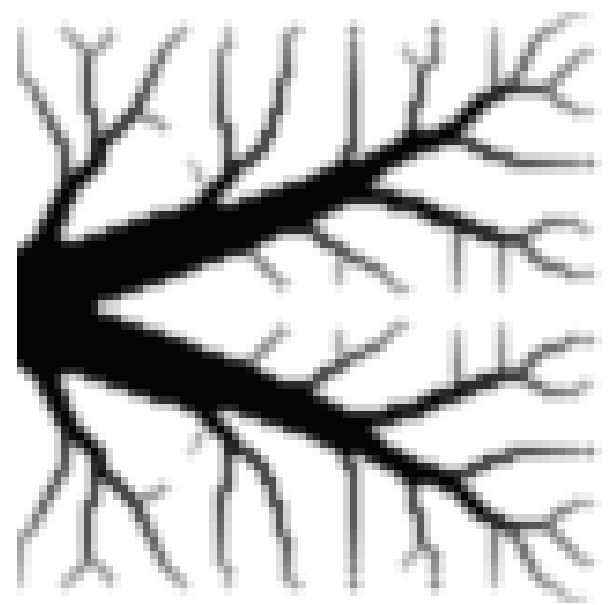

(b)

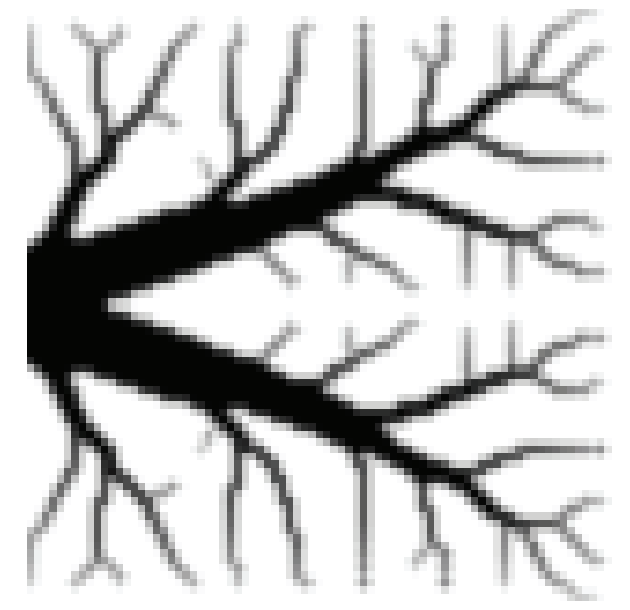

(c)

FIgure 10: Resulting topologies: (a) DTO; (b) PMA; (c) SORA.

TABLE 7: Comparison of topology optimization design results.

\begin{tabular}{lcccc}
\hline Approach & Volume/volume fraction $\left(\mathrm{mm}^{2}\right) / \%$ & Reliability index $(\beta)$ & Computing time $(\mathrm{s})$ & $\begin{array}{c}\text { Design point } \\
\mathrm{Q} / \mathrm{W} / \mathrm{mm}^{2}\end{array}$ \\
\hline DTO & $1112.7409 / 17.39$ & - & 144.75 & - \\
PMA & $1551.0111 / 24.23$ & 3.003 & 2697.55 & - \\
SORA & $1551.0111 / 24.23$ & 3.003 & 400.45 & 0.332 \\
\hline
\end{tabular}

TABLE 8: Approximated limit state function constructed by SRSM in PMA.

\begin{tabular}{lcccccc}
\hline Iteration & $a_{0}$ & $a_{1}$ & $a_{2}$ & $a_{3}$ & $a_{4}$ \\
\hline 1 & -0.6505 & 0.1861 & -0.3425 & -0.0188 & 0.0356 & -0.0174 \\
2 & -0.1788 & 0.1329 & -0.2446 & -0.0134 & 0.0254 & -0.0124 \\
$\vdots$ & $\vdots$ & $\vdots$ & $\vdots$ & $\vdots$ & $\vdots$ \\
203 & 0.3762 & 0.0703 & -0.1294 & -0.0071 & 0.0135 & -0.0066 \\
204 & 0.3762 & 0.0703 & -0.1294 & -0.0071 & 0.0135 \\
\hline
\end{tabular}

Approximated limit state function: $g(\mathbf{u})=a_{0}+a_{1} u_{1}+a_{2} u_{2}+a_{3}\left(u_{1}^{2}-1\right)+a_{4} u_{1} u_{2}+a_{5}\left(u_{2}^{2}-1\right)$. 


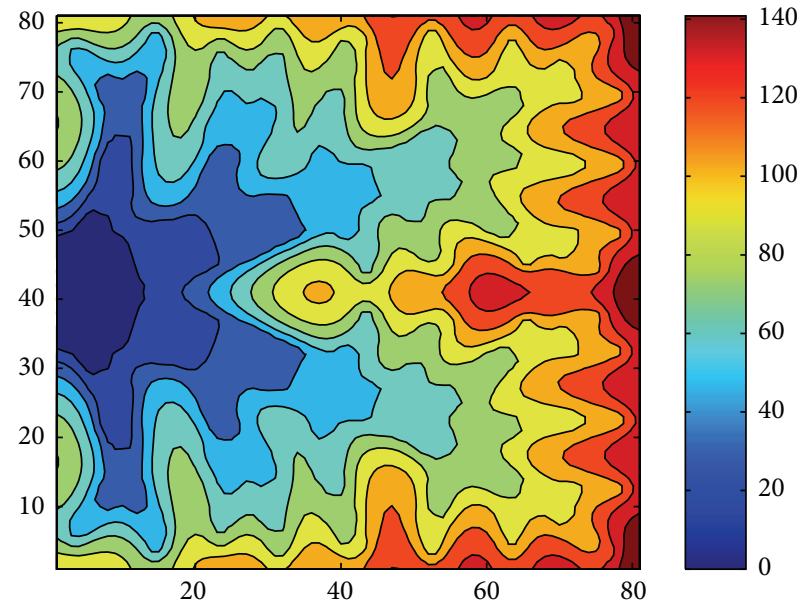

(a)

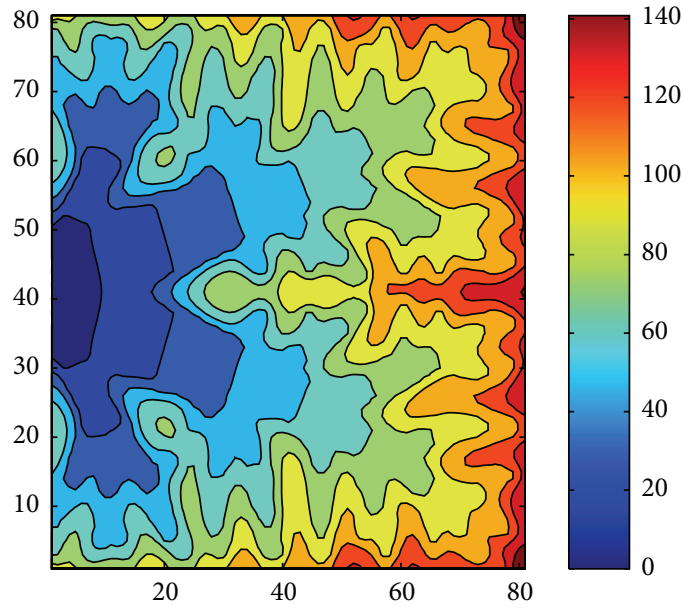

(b)

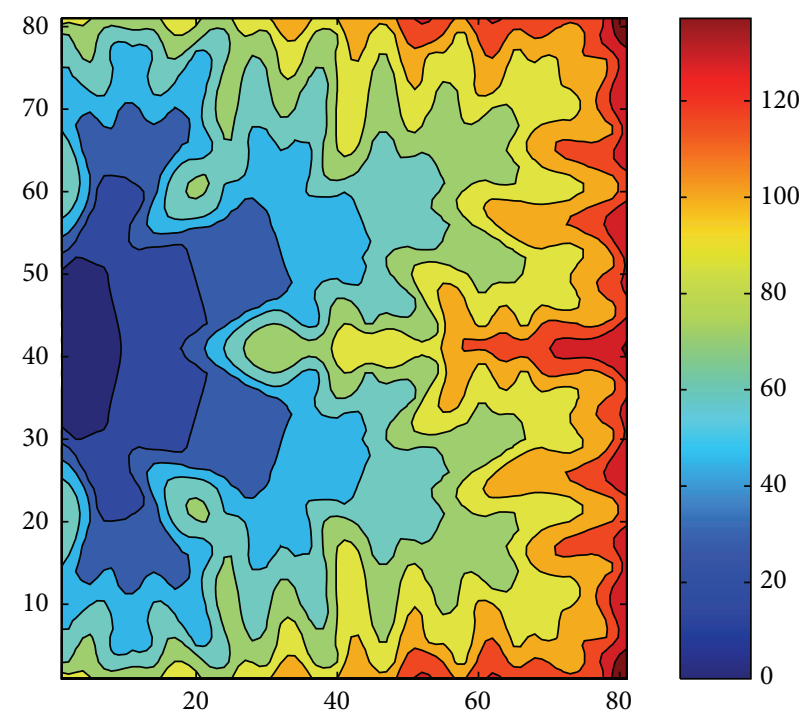

(c)

FIGURE 11: Resulting temperature contour: (a) DTO; (b) PMA; (c) SORA.

TABLE 9: Approximated limit state function constructed by SRSM in PMA.

\begin{tabular}{lcccccc}
\hline Iteration & $a_{0}$ & $a_{1}$ & $a_{2}$ & $a_{3}$ & $a_{4}$ & $a_{5}$ \\
\hline 1 & -0.0182 & 0.1148 & -0.2113 & -0.0116 & 0.0220 & -0.0107 \\
2 & 0.3762 & 0.0703 & -0.1294 & -0.0071 & 0.0135 & -0.0066 \\
\hline
\end{tabular}

\section{Conclusion}

This paper presents a mathematical framework that integrates the reliability concept into topology optimization to solve RBTO problems under uncertainty. Typically, two RBTO methodologies are implemented on several numerical examples including PMA and SORA. To enhance the computational efficiency, reliability analysis is carried out by SRSM and SGD. From the comparison of the numerical results, the following conclusions are obtained.
(1) SRSM with SGD can produce an efficient reliability analysis in RBTO, where the limit state function is approximated by SRSM, combined with SGD to generate the reasonable sampling points.

(2) The optimal solutions of RBTO are more reliable than those obtained by DTO, where more material is used and different topologies may be obtained. SORA seems to be more efficient than PMA, as the number of reliability analyses significantly reduces.

(3) It should be noticed that the computation cost of RBTO is still a challenge, because repeated reliability analysis is required for different sets of variables, especially when involving complex practical structures. One possible extension is integrating more efficient surrogate models and reasonable DOE to improve the numerical efficiency of reliability analysis. 


\section{Conflict of Interests}

The authors declare that there is no conflict of interests regarding the publication of this paper.

\section{Acknowledgments}

This research is supported by National Natural Science Foundation of China (Grant nos. 51275040 and 50905017) and the Programme of Introducing Talents of Discipline to Universities (no. B12022). The authors are also grateful to Krister Svanberg for providing his implementation of the MMA algorithm.

\section{References}

[1] M. P. Bendsøe and N. Kikuchi, "Generating optimal topologies in structural design using a homogenization method," Computer Methods in Applied Mechanics and Engineering, vol. 71, no. 2, pp. 197-224, 1988.

[2] H. A. Eschenauer and N. Olhoff, "Topology optimization of continuum structures: a review," Applied Mechanics Reviews, vol. 54, no. 4, pp. 331-389, 2001.

[3] M. P. Bendsøe and O. S. Sigmund, Topology Optimization: Theory, Methods and Application, Springer, Berlin, Germany, 2003.

[4] Y. M. Xie and G. P. Steven, Evolutionary Structural Optimization, Springer, London, UK, 1997.

[5] K. Maute and D. M. Frangopol, "Reliability-based design of MEMS mechanisms by topology optimization," Computers and Structures, vol. 81, no. 8-11, pp. 813-824, 2003.

[6] Z. Kang and Y. Luo, "Non-probabilistic reliability-based topology optimization of geometrically nonlinear structures using convex models," Computer Methods in Applied Mechanics and Engineering, vol. 198, no. 41-44, pp. 3228-3238, 2009.

[7] X. M. Zhang and G. F. Ouyang, "A level set method for reliability-based topology optimization of compliant mechanisms," Science in China E: Technological Sciences, vol. 51, no. 4, pp. 443-455, 2008

[8] K.-H. Cho, J.-Y. Park, M.-G. Im, and S.-Y. Han, "Reliabilitybased topology optimization of electro thermal-compliant mechanisms with a new material mixing method," International Journal of Precision Engineering and Manufacturing, vol. 13, no. 5, pp. 693-699, 2012.

[9] K.-H. Cho, J.-Y. Park, S.-P. Ryu, and S.-Y. Han, "Reliabilitybased topology optimization based on bidirectional evolutionary structural optimization using multi-objective sensitivity numbers," International Journal of Automotive Technology, vol. 12, no. 6, pp. 849-856, 2011.

[10] R. Y. Rubinstein, Simulation and the Monte Carlo Method, John Wiley \& Sons, New York, NY, USA, 1981.

[11] C. Sundararajan, Probabilistic Structural Mechanics Handbook: Theory and Industrial Applications, Springer, New York, NY, USA, 1995.

[12] M. R. Rajashekhar and B. R. Ellingwood, "A new look at the response surface approach for reliability analysis," Structural Safety, vol. 12, no. 3, pp. 205-220, 1993.
[13] S. S. Isukapalli, A. Roy, and P. G. Georgopoulos, "Stochastic response surface methods (SRSMs) for uncertainty propagation: application to environmental and biological systems," Risk Analysis, vol. 18, no. 3, pp. 351-363, 1998.

[14] I. Enevoldsen and J. D. Sørensen, "Reliability-based optimization in structural engineering," Structural Safety, vol. 15, no. 3, pp. 169-196, 1994.

[15] J. Tu, K. K. Choi, and Y. H. Park, "A new study on reliabilitybased design optimization," Journal of Mechanical Design (ASME), vol. 121, no. 4, pp. 557-564, 1999.

[16] C. Kim, S. Wang, K.-R. Bae, H. Moon, and K. K. Choi, "Reliability-based topology optimization with uncertainties," Journal of Mechanical Science and Technology, vol. 20, no. 4, pp. 494-504, 2006.

[17] G. Kharmanda, N. Olhoff, A. Mohamed, and M. Lemaire, "Reliability-based topology optimization," Structural and Multidisciplinary Optimization, vol. 26, no. 5, pp. 295-307, 2004.

[18] Z. Luo, L. P. Chen, J. Z. Yang, Y. Q. Zhang, and K. Abdel-Malek, "Fuzzy tolerance multilevel approach for structural topology optimization," Computers \& Structures, vol. 84, no. 3-4, pp. 127140, 2006.

[19] Y. Luo, Z. Kang, Z. Luo, and A. Li, "Continuum topology optimization with non-probabilistic reliability constraints based on multi-ellipsoid convex model," Structural and Multidisciplinary Optimization, vol. 39, no. 3, pp. 297-310, 2009.

[20] M. Silva, D. A. Tortorelli, J. A. Norato, C. Ha, and H. R. Bae, "Component and system reliability-based topology optimization using a single-loop method," Structural and Multidisciplinary Optimization, vol. 41, no. 1, pp. 87-106, 2010.

[21] T. H. Nguyen, J. Song, and G. H. Paulino, "Single-loop system reliability-based topology optimization considering statistical dependence between limit-states," Structural and Multidisciplinary Optimization, vol. 44, no. 5, pp. 593-611, 2011.

[22] K.-S. Yoo, Y.-S. Eom, J.-Y. Park, M.-G. Im, and S.-Y. Han, "Reliability-based topology optimization using successive standard response surface method," Finite Elements in Analysis and Design, vol. 47, no. 7, pp. 843-849, 2011.

[23] Y.-S. Eom, K.-S. Yoo, J.-Y. Park, and S.-Y. Han, "Reliabilitybased topology optimization using a standard response surface method for three-dimensional structures," Structural and Multidisciplinary Optimization, vol. 43, no. 2, pp. 287-295, 2011.

[24] A. M. Hasofer and N. C. Lind, "An exact and invariant first order reliability format," ASCE Journal of Engineering Mechanics, vol. 100, no. 1, pp. 111-121, 1974.

[25] X. Du and W. Chen, "Sequential optimization and reliability assessment method for efficient probabilistic design," Journal of Mechanical Design, Transactions of the ASME, vol. 126, no. 2, pp. 225-233, 2004.

[26] F. Xiong, W. Chen, Y. Xiong, and S. Yang, "Weighted stochastic response surface method considering sample weights," Structural and Multidisciplinary Optimization, vol. 43, no. 6, pp. 837849, 2011.

[27] S. A. Smolyak, "Quadrature and interpolation formulas for tensor products of certain classes of functions," Soviet Mathematics, vol. 1, no. 4, pp. 240-243, 1963.

[28] H. Yserentant, "On the multilevel splitting of finite element spaces," Numerische Mathematik, vol. 49, no. 4, pp. 379-412, 1986.

[29] T. Gerstner and M. Griebel, "Numerical integration using sparse grids," Numerical Algorithms, vol. 18, no. 3-4, pp. 209-232, 1998. 
[30] K. Svanberg, "The method of moving asymptotes-a new method for structural optimization," International Journal for Numerical Methods in Engineering, vol. 24, no. 2, pp. 359-373, 1987.

[31] O. Sigmund, "Morphology-based black and white filters for topologyoptimization," Structural and Multidisciplinary Optimization, vol. 33, no. 4-5, pp. 401-424, 2007. 


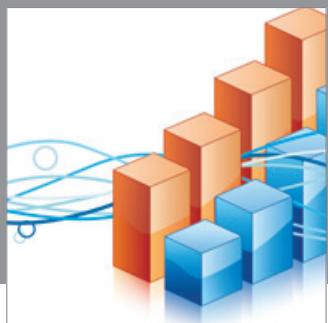

Advances in

Operations Research

mansans

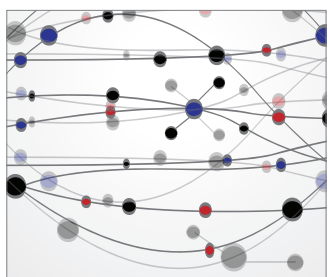

The Scientific World Journal
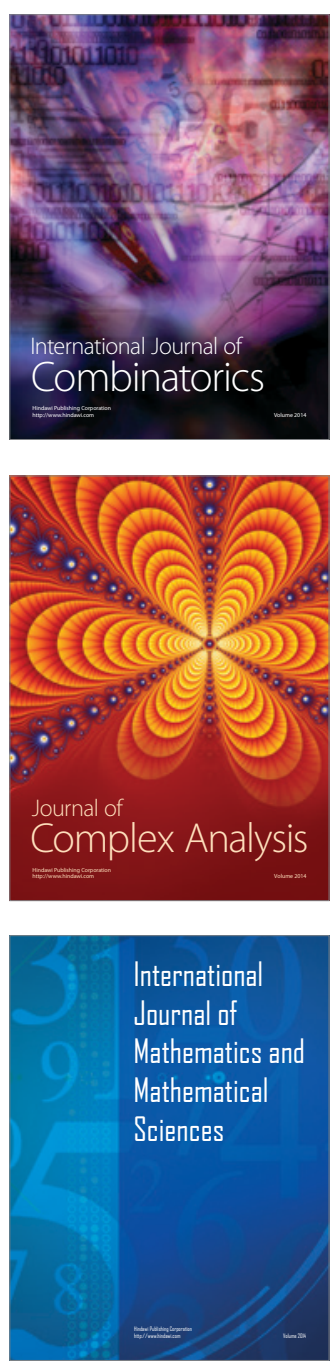
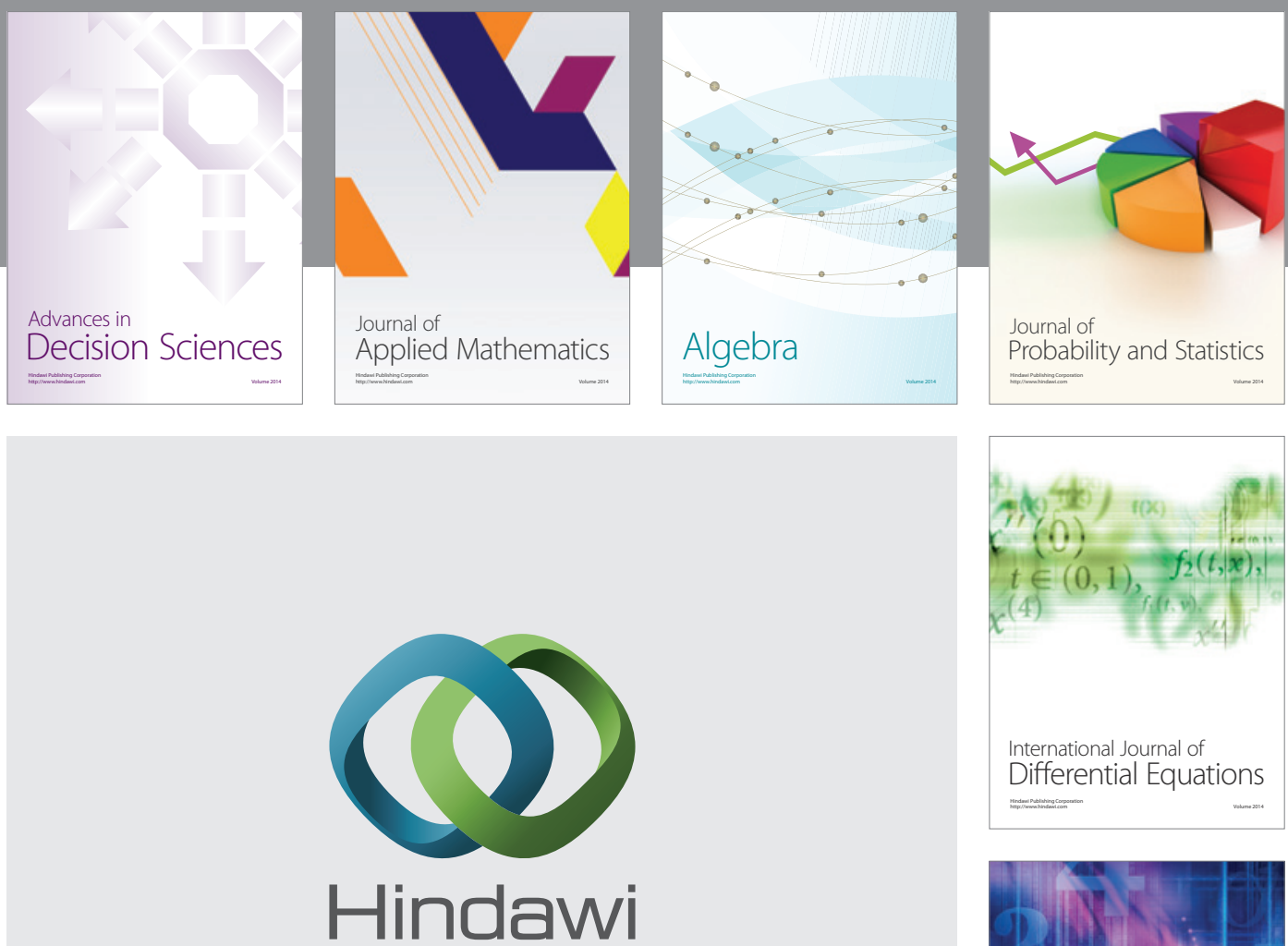

Submit your manuscripts at http://www.hindawi.com
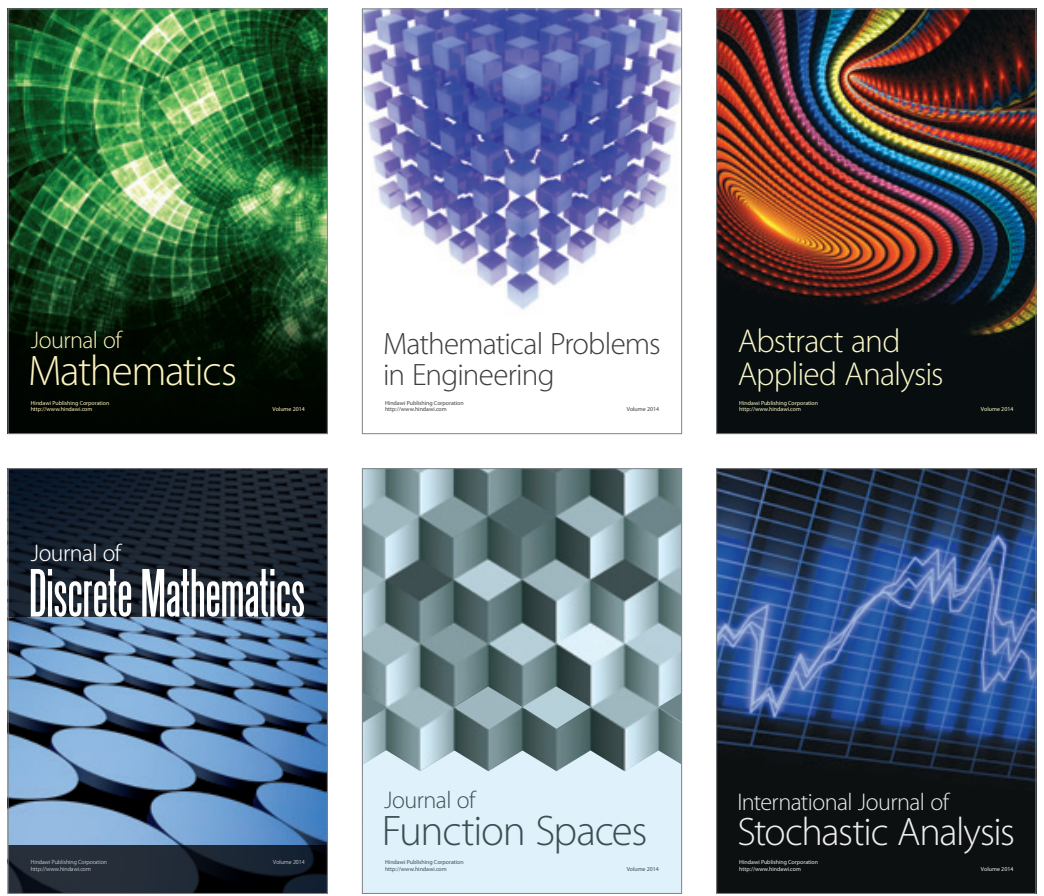

Journal of

Function Spaces

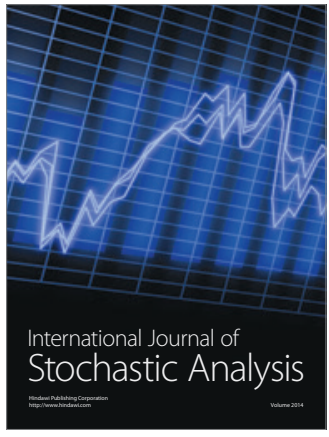

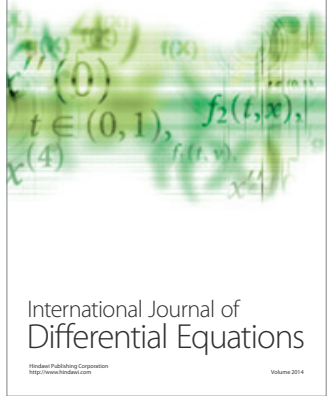
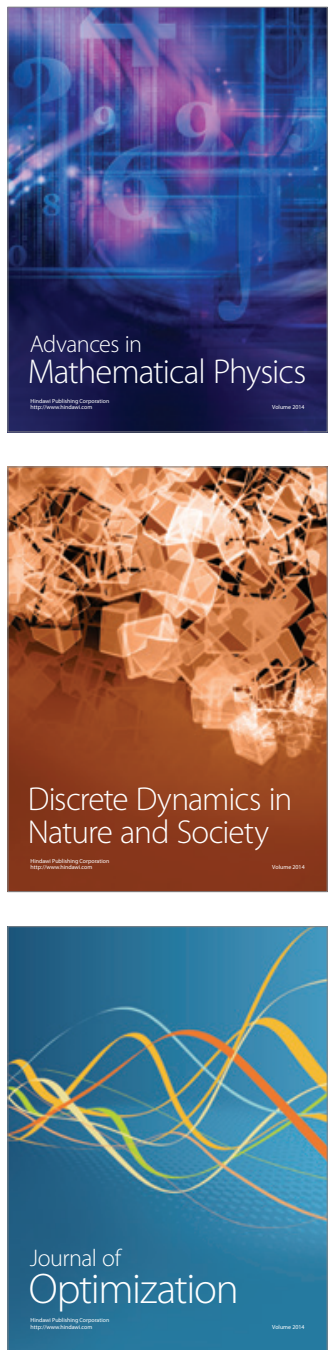